\section{Spontaneous external drainage of perinephric abscess in spinal cord injury patients through previous nephrostomy track}

In a recent issue of Spinal Cord, Deck and Yang ${ }^{1}$ discussed the management of perinephric abscess in three patients with spinal cord injury. All patients required operative drainage of perinephric abscess and the ipsilateral kidney was nonfunctioning or poorly functioning. We report two spinal cord injury patients in whom perinephric abscess drained to the exterior spontaneously through a previous nephrostomy track. These patients had undergone nephrostomy in the past and the nephrostomy track had healed completely after removal of the nephrostomy catheter. Spontaneous external drainage of pus through a nephrostomy track saved these patients from developing major complications such as loss of function in the ipsilateral kidney, systemic sepsis, or rupture of perinephric abscess in to adjacent structures with potentially serious consequences. ${ }^{2}$

\section{Case 1}

This patient sustained T4 complete paraplegia in a motorbike accident 13 years ago when he was 17 years old. Seven years after spinal cord injury, he developed bilateral pyonephrosis. There was a ureteropelvic junction obstruction on the right side. On the left side, there were ureteric and renal calculi. Both kidneys were drained by percutaneous 8-French nephrostomy. Left ureteroscopy and electrohydraulic lithotripsy of ureteric stone were carried out. Subsequently, the left renal stone was treated by extra corporeal shock wave lithotripsy. He underwent pyeloplasty on the right side. The nephrostomy tubes were removed and he managed the bladder by intermittent

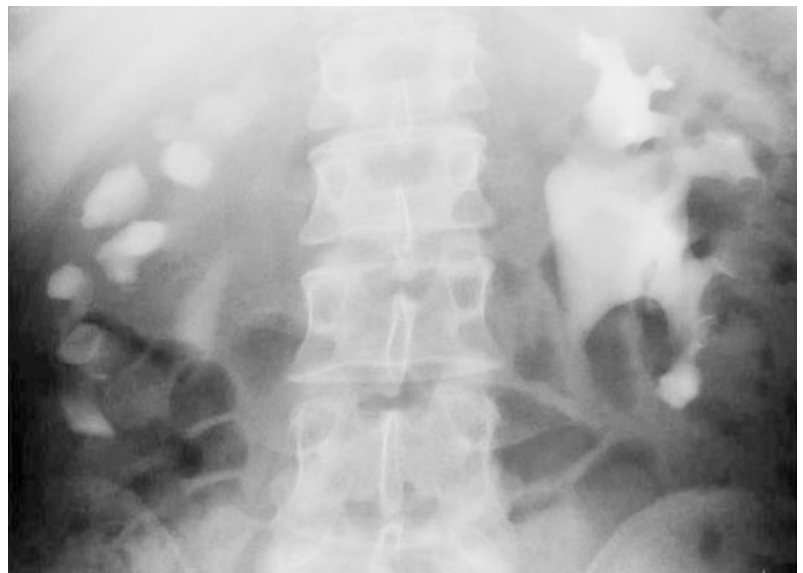

Figure 1 Case 1: 10-min IVU film (30082000) shows good excretion of contrast by left kidney with normal pelvicalyceal system. There is delayed excretion by the right kidney with caliectasis catheterisation. He was in good health for 2 years. Then he started developing urinary infections and became unwell. A few days later, there was spontaneous discharge of pus from the left nephrostomy scar. Intravenous urography (IVU) showed prompt excretion of contrast by the left kidney with normal pelvicalyceal system. Right kidney showed delayed excretion of contrast; there was dilation of renal pelvis and calyces (Figure 1). Computerised tomography (CT) of the abdomen revealed an abscess measuring $5 \times 4-\mathrm{cm}$ located posterior to the left kidney (Figure 2). Microbiology of the pus yielded a heavy growth of mixed anaerobes sensitive to metronidazole. He was prescribed metronidazole intravenously. The nephrostomy track was kept open to allow drainage of pus. Pus discharge from the nephrostomy scar continued for 4 weeks. A CT scan, performed 4 weeks after the initial CT scan, showed marked resolution of the left perinephric abscess (Figure 3). A follow-

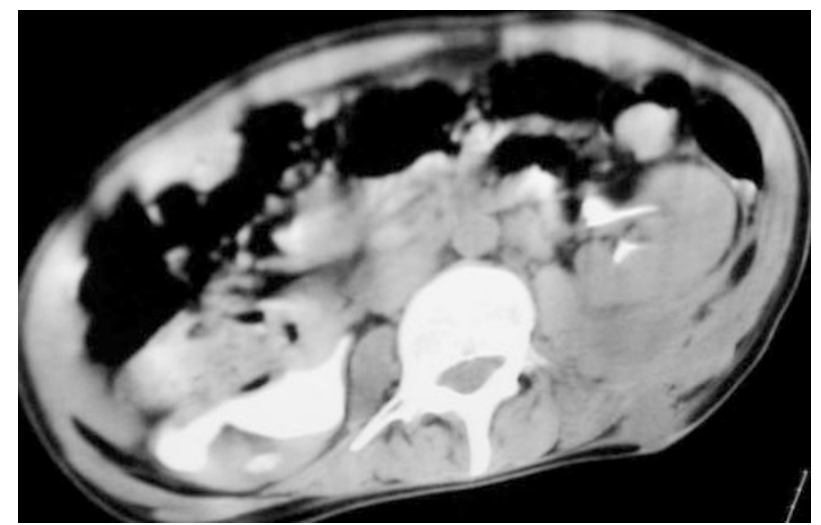

Figure 2 Case 1: CT of abdomen (30082000) shows an abscess measuring $5 \times 4-\mathrm{cm}$ located posterior to left kidney

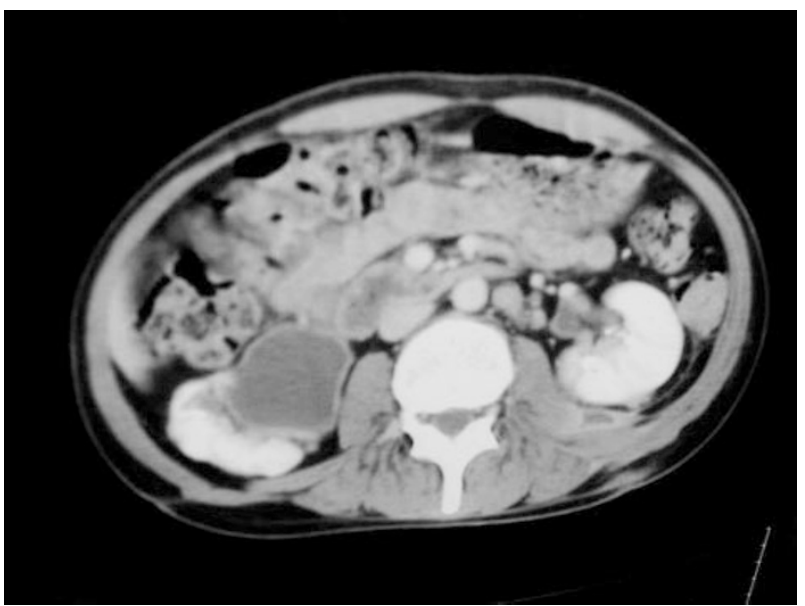

Figure 3 Case 1: CT of abdomen (02102000) shows marked resolution of left perinephric abscess 


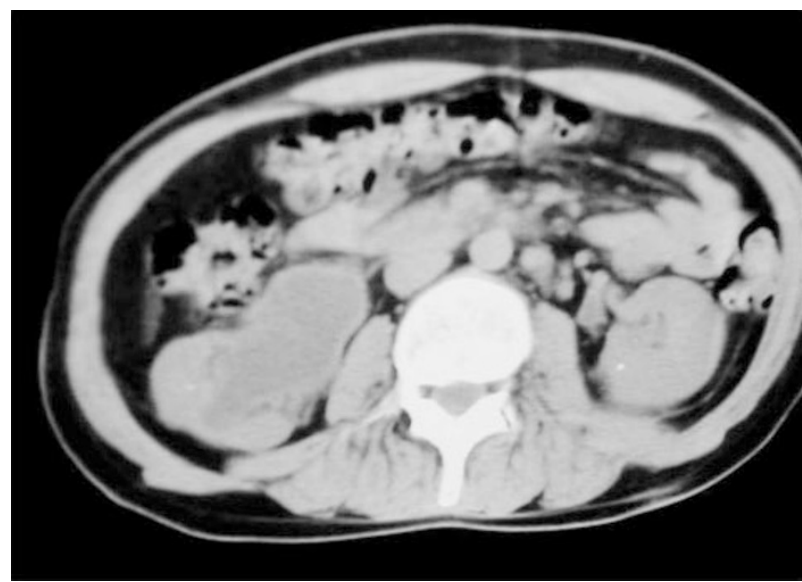

Figure 4 Case 1: CT of abdomen (05122000) shows complete resolution of left perinephric abscess

up CT scan, performed 9 weeks later, showed complete resolution of perinephric abscess (Figure 4). With cessation of pus discharge, the nephrostomy track healed completely.

\section{Case 2}

This male patient sustained tetraplegia in a motor bike accident when he was 18 years old. He underwent right percutaneous nephrostolithotomy twice (14 and 16 years after the initial accident). Right pyelolithotomy and nephrostomy with a 16French Foley catheter were carried out 24 years after he had sustained tetraplegia. The nephrostomy site had healed completely after removal of nephrostomy catheter. Two years later, he developed a lump in the right loin, which burst open at the site of previous nephrostomy, discharging pus. Ultrasound scan of right kidney showed small calculi in the renal pelvis and lower pole, where scarring was seen. The sinus in the right loin communicated via an oblique tract to a $3-\mathrm{cm}$ perinephric collection abutting the lower pole of the right kidney (Figure $5 \mathrm{~A}$ and $\mathrm{B}$ ). Microbiology of the pus yielded micro-aerophilic, gram-negative bacillus sensitive to imipenem. He was initially prescribed ciprofloxacin and then meropenem. The nephrostomy track was kept open to facilitate the drainage of pus, which continued to discharge for about 10 days. CT of the abdomen, performed 2 weeks later, showed complete resolution of perinephric abscess. Following cessation of pus discharge, the nephrostomy track healed well.

\section{Discussion}

These cases illustrate that a previous nephrostomy track can open up even after several months, to provide an exit path for perinephric abscess to drain to the external surface. Thus an old nephrostomy track, which appears to be closed and well healed for all intents and purposes, may act as a safety vent for spontaneous external drainage of pus from the perinephric region. By providing a passage for drainage of pus to the external surface, the nephrostomy track helped to prevent complications of perinephric abscess such as systemic sepsis and deterioration of ipsilateral renal function, which could ultimately, require nephrectomy. In both cases, spontaneous drainage of pus through a previous nephrostomy track, and prolonged antibiotic therapy based on microbiology report of pus, played vital
A
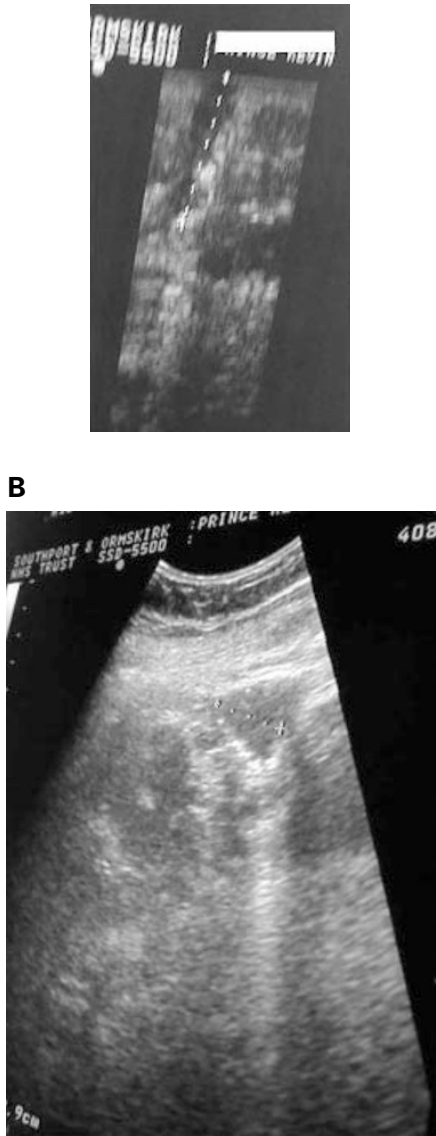

Figure 5 Case 2: Ultrasound scan (13072001) shows the sinus in the right loin (A) communicating via an oblique tract to a $3-\mathrm{cm}$ perinephric collection abutting the lower pole of right kidney $(\mathbf{B})$

roles in preservation of ipsilateral renal function and achieving complete resolution of perinephric abscess.

S Vaidyanathan, BM Soni, T Oo, G Singh Regional Spinal Injuries Centre, District General Hospital, Southport, Merseyside, PR8 6PN, UK

P Hughes

Department of Radiology, District General Hospital, Southport, Merseyside, PR8 6PN, UK

\section{References}

1 Deck AJ, Yang CC. Perinephric abscesses in the neurologically impaired. Spinal Cord 2001; 39: 477-481.

2 Vaidyanathan S et al. Silent hydronephrosis/pyonephrosis due to upper urinary tract calculi in spinal cord injury patients. Spinal Cord 2000; 38: $661-668$. 